\title{
Distributed Rotating Encirclement Control of Strict-Feedback Multi-Agent Systems Using Bearing Measurements
}

\author{
Tengfei Zhang, Yingmin Jia* \\ The Seventh Research Division and the Center for Information and Control, School of Automation Science and \\ Electrical Engineering, Beihang University (BUAA), 37 Xueyuan Road, Haidian District, Beijing, 100191, China \\ E-mail:tfzhang@buaa.edu.cn,ymjia@buaa.edu.cn \\ www.buaa.edu.cn
}

\begin{abstract}
This paper investigates the distributed multi-target rotating encirclement formation problem of strict-feedback multiagent systems using the targets' bearing angles and the agents' known positions, where all agents are forced to achieve even circular formation around the targets' geometric center. Firstly, an estimator is proposed for each agent to localize the neighbor targets. Secondly, based on the trajectory planning method, a reference trajectory is constructed by three estimators, which are used to obtain the targets' geometric center, the reference rotating radius and angular. Then, the proposed adaptive neural dynamic surface control law forces each agent to move along the reference trajectory, which satisfies the multi-target rotating encirclement formation conditions.
\end{abstract}

Keywords: Strict-feedback multi-agent systems, rotating encirclement control, target localization, trajectory planning, trajectory tracking

\section{Introduction}

Recent years the rotating encirclement formation problem of multi-agent systems have attracted considerable attention due to its significant potential applications in both military and civilian areas such as surveillance, search-and-rescue, reconnaissance, etc ${ }^{1}$. Many interesting results have been achieved for the rotating formation or surrounding/encirclement control problem ${ }^{1-7}$.

As one of the most important high-order systems, the strict-feedback system can be used to model a variety of physical systems including robotic manipulators, vessel, unmanned aerial vehicle and so on $^{8}$. And recent years have witnessed the emergence of researches with respect to the strict-feedback single/multi-agent system ${ }^{8-11}$. However, there is no research to date on the rotating encirclement control of high-order multi-agent system.
Motivated by above discussion, for the first time, we consider the multi-target rotating encirclement formation problem of strict-feedback multi-agent systems, and only bearing measurements of targets can be obtained. To this end, we divide the problem into three subproblems: target localization, trajectory planning and trajectory tracking. Four estimators are designed to construct a reference trajectory for each agent, and an adaptive neural dynamic surface control law is proposed to make the agent move along the desired trajectory.

\section{Preliminaries and Problem Statement}

\subsection{Graph Theory}

Let $\mathcal{G}(\mathcal{V}, \mathcal{E}, \mathcal{A}, \mathcal{B})$ be a weighted undirected graph corresponding to $n$ agents and $m$ targets, where $\mathcal{V}=$ $\left\{v_{1}, v_{2}, \cdots, v_{n}, s_{1}, \cdots, s_{m}\right\}$ denotes the set of vertexes, $\mathcal{E} \subset$

${ }^{*}$ Corresponding author.

(C) The 2020 International Conference on Artificial Life and Robotics (ICAROB2020), Jan. 13-16, B-Con Plaza, Beppu, Oita, Japan 
$\mathcal{V} \times \mathcal{V}$ denotes the set of edges, $\mathcal{A}=\left[a_{i j}\right] \in \mathrm{R}^{n \times n}$ denotes the weighted adjacency matrix of targets, $\mathcal{B}=\left[b_{i k}\right] \in$ $\mathrm{R}^{n \times m}$ denotes the weighted adjacency matrix from targets to agents. Let $d\left(v_{i}, v_{j}\right)$ denote the shortest distance from the vertex $v_{i}$ to $v_{j}$, for instance, $d\left(v_{i}, v_{j}\right)=1$ if $\left(v_{i}, v_{j}\right) \in$ $\mathcal{E}$. The neighbor agents set of the agent $v_{i}$ is denoted by $\mathcal{N}_{i}=\left\{v_{j} \in \mathcal{V} \mid\left(v_{i}, v_{j}\right) \in \mathcal{E}\right\}$ and the neighbor targets set of the agent $v_{i}$ is denoted by $\mathcal{N}_{i}^{\mathcal{T}}=\left\{s_{k} \in \mathcal{V} \mid\left(v_{i}, s_{k}\right) \in \mathcal{E}\right\}$. The neighbor agents set of the target $s_{k}$ is denoted by $\mathcal{N}_{k}^{\mathcal{J}}=\left\{v_{i} \in \mathcal{V} \mid\left(v_{i}, s_{k}\right) \in \mathcal{E}\right\}$. And let $|\cdot|$ denote the number of elements in the set .

\subsection{Problem Statement}

Consider a multi-agent system consisting of $n$ agents (Index set $\mathcal{J}=\{1,2, \cdots, n\}$ ) and $m$ stationary targets (Index set $\mathcal{T}=\{1,2, \cdots, m\}$ ) with bearing-only measurements, where the dynamic of agent $v_{i}$ is in the following $q_{i}$-order strict-feedback form.

$$
\left\{\begin{array}{l}
\dot{x}_{i j}=f_{i j}\left(\bar{x}_{i j}\right)+x_{i j+1} \\
\dot{x}_{i q_{i}}=f_{i_{q_{i}}}\left(\bar{x}_{i q_{i}}\right)+u_{i} \\
y_{i}=x_{i 1}
\end{array}\right.
$$

where $\bar{x}_{i j}=\left[x_{i 1}^{\mathrm{T}}, \cdots, x_{i j}^{\mathrm{T}}\right]^{\mathrm{T}}$, and $\bar{x}_{i q_{i}}, y_{i}, u_{i} \in \mathrm{R}^{2}$ represent the states, output and control input of agent $v_{i}$, respectively. $f_{i j}\left(\bar{x}_{i j}\right)$ is an unknown continuous nonlinear function.

The objective of this note is to design the distributed control scheme using bearing-only measurements of targets and the neighbor position information of agents, such that strict-feedback agents are capable of achieving the multi-target rotating encirclement formation, which is properly formulated by Definition 1 using the polar coordinate transformation using the polar coordinate transformation $y_{i}=\bar{r}+\left[l_{i} \cos \left(\theta_{i}\right), l_{i} \sin \left(\theta_{i}\right)\right]^{\mathrm{T}}$.

Definition $\mathbf{1}^{\mathbf{5}}$. The multi-agent system is said to achieve the multi-target rotating encirclement formation if

$$
\begin{aligned}
& \lim _{t \rightarrow \infty}\left[l_{i}-\lambda \max _{k \in \mathcal{T}}\left\|r_{k}-\bar{r}\right\|\right]=0 \\
& \lim _{t \rightarrow \infty}\left[\theta_{i}-\theta_{j}-\frac{2 \pi(i-j)}{n}\right]=0 \\
& \lim _{t \rightarrow \infty}\left[\dot{\theta}_{i}-\omega\right]=0
\end{aligned}
$$

Where $i, j \in \mathcal{J}, r_{k}$ and $\bar{r}(t)=1 / m \sum_{k \in \mathcal{T}} r_{k}$ denote the position of the $k$-th target and the geometric center of all targets respectively. The design parameter $\lambda>1$ determines the radius of the desired rotation formation. And $\omega$ is the desired angular velocity.

To facilitate the later control design and analysis, we make some reasonable assumptions.
Assumption 1. All agents are connected in some undirected communication topologies and each target connectes to at least one agent via the directed edge.

Assumption 2. The radius of the desired rotation formation is bounded, i.e., there exists a positive constant $d^{*}$ satisfying $\max _{k \in \mathcal{T}}\left\|r_{k}-\bar{r}\right\| \leq d^{*}$.

Assumption 3. The desired angular velocity $\omega$ and angular acceleration $\dot{\omega}$ are continuous and bounded, i.e., there exists positive constants $\omega^{*}$ and $\omega_{d}^{*}$ such that $\|\omega\| \leq \omega^{*},\|\dot{\omega}\| \leq \omega_{d}^{*}$.

\section{Control Design}

In this section we present in detail the distributed multitarget rotating encirclement control scheme, which includes three parts: target localization, trajectory planning and trajectory tracking.

\subsection{Target Localization}

To estimate the neighbor target's position of agent $v_{i}$ with bearing-only measurements, the following estimator is proposed according to Ref. 7.

$$
\dot{\hat{r}}_{i k}=\alpha_{i k}\left(I-\varphi_{i k} \varphi_{i k}^{\mathrm{T}}\right)\left(x_{i 1}-\hat{r}_{i k}\right)
$$

Where $k \in \mathcal{N}_{i}^{\mathcal{T}}, \alpha_{i k}$ is a positive design parameter, and $\varphi_{i k}$ is the unit vector from $x_{i}$ to $r_{k}$, i.e., $\varphi_{i k}=\left(r_{k}-\right.$ $\left.x_{i}\right) /\left\|r_{k}-x_{i}\right\|$.

\subsection{Trajectory Planning}

For each agent $v_{i}$, to plan a reference trajectory satisfying the Definition 1, we design the following distributed estimators to obtain the estimations $p_{i}, \hat{l}_{i}$ and $\hat{\theta}_{i}$ of the desired geometric center $\bar{r}$, polar radius $l_{i}$ and polar angle $\theta_{i}$, respectively.

$$
\begin{aligned}
& \left\{\begin{array}{c}
\dot{p}_{i k}=\beta_{i} \sum_{j \in \mathcal{N}_{i}} a_{i j}\left[p_{j k}-p_{i k}\right] \\
\quad+\beta_{i} b_{i k}\left[\hat{r}_{i k}-p_{i k}\right] \\
p_{i}=\frac{1}{m} \sum_{k=1}^{m} p_{i k}
\end{array}\right. \\
& \left\{\begin{array}{c}
\dot{\rho}_{i 1}=\gamma_{i 1} \max _{k \in \mathcal{N}_{i}^{T}}\left(\left\|\hat{r}_{i k}-p_{i}\right\|\right)-\rho_{i 1} \\
\dot{\rho}_{i 2}=\gamma_{i 2} \max _{j \in \mathcal{N}_{i} \cup\{i\}}\left(\left\|\rho_{j 1}\right\|\right)-\rho_{i 2} \\
\vdots \\
\dot{\rho}_{i M}=\gamma_{i M} \max _{j \in \mathcal{N}_{i} \cup\{i\}}\left(\left\|\rho_{j M-1}\right\|\right)-\rho_{i M} \\
\hat{l}_{i}=\lambda \rho_{i M} \quad \\
\dot{\hat{\theta}}_{i}=\delta_{i} \sum_{j \in \mathcal{N}_{i}} a_{i k}\left[\hat{\theta}_{j}-\hat{\theta}_{i}-\frac{2 \pi(j-i)}{n}\right]+\omega
\end{array}\right.
\end{aligned}
$$

Where $\beta_{i}, \gamma_{i 1}, \cdots, \gamma_{i M}, \delta_{i}$ are positive design parameters, and $M=\max _{i, j \in \mathcal{J}}\{d(i, j)\}$, which can be chosen as $M=$ $n-1$ if it is not prior information.

Then, with the polar coordinate transformation, the reference trajectory of agent $v_{i}$ is provided as follows. 


$$
\hat{y}_{i}=p_{i}+\left[\hat{l}_{i} \cos \left(\hat{\theta}_{i}\right), \hat{l}_{i} \sin \left(\hat{\theta}_{i}\right)\right]^{\mathrm{T}}
$$

\subsection{Trajectory Tracking}

Similar to the backstepping-based DSC design procedure, we define dynamic surface errors as follows.

$$
\left\{\begin{array}{c}
z_{i 1}=x_{i 1}-\hat{y}_{i} \\
z_{i j}=x_{i j}-\hat{\eta}_{i j}
\end{array}\right.
$$

Where $\hat{\eta}_{i j}(t)$ is the first-order filter estimation of the virtual controller $\eta_{i j}(t)$ with the time constant $\tau_{i j}>0$ and the filter error is denoted by $\tilde{\eta}_{i j}=\hat{\eta}_{i j}-\eta_{i j}$.

$$
\tau_{i j} \dot{\hat{\eta}}_{i j}+\hat{\eta}_{i j}=\eta_{i j}, \hat{\eta}_{i j}(0)=\eta_{i j}(0)
$$

Then, we will present the following virtual and actual controllers and adaptive law such that each agent moves along its desired reference trajectory.

$$
\begin{aligned}
& \left\{\begin{array}{l}
\eta_{i j}=-\kappa_{i j} z_{i j}-\widehat{W}_{i j}^{\mathrm{T}} S_{i j}\left(\zeta_{i j}\right) \\
u_{i}=-\kappa_{i q_{i}} z_{i q_{i}}-\widehat{W}_{i q_{i}}^{\mathrm{T}} S_{i q_{i}}\left(\zeta_{i q_{i}}\right)
\end{array}\right. \\
& \dot{\widehat{W}}_{i j}=-\Gamma_{i j}^{-1}\left[\sigma_{i j} \widehat{W}_{i j}-S_{i j}\left(\zeta_{i j}\right) z_{i j}^{\mathrm{T}}\right]
\end{aligned}
$$

Where $\Gamma_{i j}=\Gamma_{i j}^{\mathrm{T}}>0$ is an adaptive gain matrix, $\widehat{W}_{i j}$ and $S_{i j}\left(\zeta_{i j}\right)$ represent the estimation of the optimal weight matrix $W_{i j}$ and the basis function vector respectively. And $\kappa_{i j}, \sigma_{i j}$ are positive design parameters.

\section{Main Results}

With the proposed control scheme in Section 3, we can easily obtain the following reasonable results.

Lemma 1. Consider the estimator (5) under Assumptions 1-2. Then for any $i \in \mathcal{J}, k \in \mathcal{T}$, the estimation position $\hat{r}_{i k}$ will asymptotically converge to the actual position $r_{k}$ of the $k$-th target.

Proof. The proof is similar to Theorem 3.1 in Ref. 7.

Then, we define the estimation of the targets' geometric center as follows.

$$
\overline{\hat{r}}=\frac{1}{m} \sum_{i=1}^{n} \sum_{k \in \mathcal{N}_{i}^{\mathcal{T}}} \frac{1}{\left|\mathcal{N}_{k}^{J}\right|} \hat{r}_{i k}
$$

Apparently, $\overline{\hat{r}}$ will asymptotically converge to the actual geometric center $\bar{r}$.

Lemma 2. Consider the estimator (6) under Assumptions 1-2. For any $i \in \mathcal{J}$, the estimation position $p_{i}$ will asymptotically converge to $\overline{\hat{r}}$.

Proof. The proof is similar to Lemma 4 in Ref. 5 ..

Then, combining lemma 1 with lemma 2 , we can conclude that the estimation position $p_{i}$ of the $i$-th agent will asymptotically converge to the actual geometric center $\bar{r}$. Lemma 3. Consider the estimator (7) under Assumptions 1-2. For any $i \in \mathcal{J}$, the following equation holds.

$$
\lim _{t \rightarrow \infty}\left[\hat{l}_{i}-\lambda \max _{i \in \mathcal{J}, k \in \mathcal{N}_{i}^{\mathcal{T}}}\left(\left\|\hat{r}_{i k}-p_{i}\right\|\right)\right]=0
$$

In other words, the estimation $\hat{l}_{i}$ of the polar radius will asymptotically converge to the above value.

Proof. The proof is similar to Lemma 5 in Ref. 5.

Furthermore, with Lemma 1 and Lemma 2, it is easy to see that $\lim \left[\hat{l}_{i}-\lambda \max _{k \in \mathcal{T}}\left(\left\|r_{k}-\bar{r}\right\|\right)\right]=0$, which im-

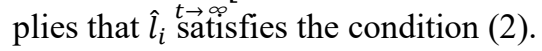

Lemma 4. Consider the estimator (8) under Assumptions 1-3. For any $i, j \in \mathcal{J}$, the following equations hold.

$$
\begin{gathered}
\lim _{t \rightarrow \infty}\left[\hat{\theta}_{i}-\hat{\theta}_{j}-\frac{2 \pi(i-j)}{n}\right]=0 \\
\lim _{t \rightarrow \infty}\left[\dot{\hat{\theta}}_{i}-\omega\right]=0
\end{gathered}
$$

In other words, the estimation $\hat{\theta}_{i}$ of the polar angle satisfies conditions (3) and (4).

Proof. The proof is similar to Lemma 6 in Ref. 5.

Thus, from the polar coordinate transformation of (9), we can easily conclude that the reference trajectory $\hat{y}_{i}$ satisfies conditions of the multi-target rotating encirclement formation in Definition 1.

Then, we will carry on the stability analysis of the proposed control scheme (12) and (13), which drives the output $y_{i}$ of the agent $v_{i}$ to the reference trajectory $\hat{y}_{i}$. Since the neural network is capable of approaching any continual nonlinear function with free precision (See Lemma 2 in Ref. 8 for details), we make the following reasonable approximation.

$$
\begin{aligned}
\phi_{i 1} & =W_{i 1}^{\mathrm{T}} S_{i 1}\left(\zeta_{i 1}\right)+\varepsilon_{i 1}\left(\zeta_{i 1}\right) \\
& =f_{i 1}\left(\bar{x}_{i 1}\right)-\dot{\hat{y}}_{i} \\
\phi_{i j} & =W_{i j}^{\mathrm{T}} S_{i j}\left(\zeta_{i j}\right)+\varepsilon_{i j}\left(\zeta_{i j}\right) \\
& =f_{i j}\left(\bar{x}_{i j}\right)+\tilde{\eta}_{i j} / \tau_{i j}+z_{i j-1}
\end{aligned}
$$

Where $\left\|\varepsilon_{i j}\left(\zeta_{i j}\right)\right\|<\varepsilon_{i j}^{*}$, $\varepsilon_{i j}^{*}$ is an arbitrarily small constant and denote $\widetilde{W}_{i j}=W_{i j}-\widehat{W}_{i j}$.

Choose a common Lyapunov function candidate as $V_{i}=\frac{1}{2} \sum_{j=1}^{q_{i}}\left[z_{i j}^{\mathrm{T}} z_{i j}+\operatorname{tr}\left(\widetilde{W}_{i j}^{\mathrm{T}} \Gamma_{i j} \widetilde{W}_{i j}\right)\right]+\frac{1}{2} \sum_{j=2}^{q_{i}} \tilde{\eta}_{i j}^{\mathrm{T}} \tilde{\eta}_{i j}$. Thus, by calculating the time derivative of $V_{i}$, we have

$$
\begin{aligned}
\dot{V}_{i}= & \sum_{j=1}^{q_{i}} z_{i j}^{\mathrm{T}}\left[-\kappa_{i j} z_{i j}+\widetilde{W}_{i j}^{\mathrm{T}} S_{i j}\left(\zeta_{i j}\right)+\varepsilon_{i j}\left(\zeta_{i j}\right)\right] \\
& +\sum_{j=2}^{q_{i}} z_{i j-1}^{\mathrm{T}} \tilde{\eta}_{i j}+\sum_{j=1}^{q_{i}} \operatorname{tr}\left(\widetilde{W}_{i j}^{\mathrm{T}} \Gamma_{i j} \dot{\widetilde{W}}_{i j}\right) \\
& +\sum_{j=2}^{q_{i}} \tilde{\eta}_{i j}^{\mathrm{T}} \dot{\tilde{\eta}}_{i j}
\end{aligned}
$$

The dynamic of the filter error $\tilde{\eta}_{i j}$ can be written as

$$
\dot{\tilde{\eta}}_{i j}=-\frac{\widetilde{\eta}_{i j}}{\tau_{i j}}+\pi_{i j}\left(\bar{z}_{i j}, \overline{\tilde{\eta}}_{i j}, \bar{W}_{i j-1}, Y_{i}\right)
$$

Where we denote $\bar{z}_{i j}=\left[z_{i 1}^{\mathrm{T}}, \cdots, z_{i j}^{\mathrm{T}}\right]^{\mathrm{T}}, \overline{\tilde{\eta}}_{i j}=\left[\tilde{\eta}_{i 2}^{\mathrm{T}}, \cdots, \tilde{\eta}_{i j}^{\mathrm{T}}\right]^{\mathrm{T}}$, $\overline{\widetilde{W}}_{i j}=\left[\widetilde{W}_{i 1}^{\mathrm{T}}, \cdots, \widetilde{W}_{i j}^{\mathrm{T}}\right]^{\mathrm{T}}$ and $Y_{i}=\left[\hat{y}_{i}^{\mathrm{T}}, \dot{\hat{y}}_{i}^{\mathrm{T}}, \ddot{\hat{y}}_{i}^{\mathrm{T}}\right]^{\mathrm{T}}$.

Therefore, substituting the adaptive law (13) and (20) into (19), we obtain

\footnotetext{
(C) The 2020 International Conference on Artificial Life and Robotics (ICAROB2020), Jan.13-16, B-Con Plaza, Beppu, Oita, Japan
} 


$$
\begin{aligned}
\dot{V}_{i}= & -\sum_{j=1}^{q_{i}} \kappa_{i j} z_{i j}^{\mathrm{T}} z_{i j}-\sum_{j=1}^{q_{i}} z_{i j}^{\mathrm{T}} \varepsilon_{i j}\left(\zeta_{i j}\right) \\
& +\sum_{j=1}^{q_{i}} \sigma_{i j} \operatorname{tr}\left(\widetilde{W}_{i j}^{\mathrm{T}} \widehat{W}_{i j}\right)-\sum_{j=2}^{q_{i}} \frac{\widetilde{\eta}_{i j}^{\mathrm{T}} \tilde{\eta}_{i j}}{\tau_{i j}} \\
& +\sum_{j=2}^{q_{i}} z_{i j-1}^{\mathrm{T}} \tilde{\eta}_{i j}+\sum_{j=2}^{q_{i}} \tilde{\eta}_{i j}^{\mathrm{T}} \pi_{i j}
\end{aligned}
$$

According to Assumption 2 and 3, we know that the desired rotating radius, angular velocity and angular acceleration are bounded. Then $\hat{y}_{i}, \dot{\hat{y}}_{i}$ and $\ddot{\hat{y}}_{i}$ are bounded, i.e., there exists a positive constant $Y_{i}^{*}$ such that $\Xi_{\mathrm{i}}=$ $\left\{Y_{i}\left\|\hat{y}_{i}\right\|+\left\|\dot{\hat{y}}_{i}\right\|+\left\|\ddot{\hat{y}}_{i}\right\| \leq Y_{i}^{*}\right\}$. In addition, we denote that $\Pi_{i}=\left\{\left(\bar{z}_{i q_{i}}, \overline{\tilde{\eta}}_{i q_{i}}, \overline{\widetilde{W}}_{i q_{i}}\right) \mid V_{i} \leq 2 \mu_{i}\right\}$, where $\mu_{i}$ is a positive constant. Then, it is not hard to see that $\Xi_{i}$ and $\Pi_{i}$ are compact sets. Thus, there exists a positive constant $\pi_{i j}^{*}$ satisfying $\left\|\pi_{i j}(\cdot)\right\| \leq \pi_{i j}^{*}$.

Moreover, with Young's inequality ${ }^{8}$, we have

$$
\left\{\begin{array}{l}
z_{i j}^{\mathrm{T}} \varepsilon_{i j}\left(\zeta_{i j}\right) \leq\left(\varepsilon_{i j}^{*} / 2 \varrho_{i j}\right)\left\|z_{i j}\right\|^{2}+\varrho_{i j} / 2 \\
\operatorname{tr}\left(\widetilde{W}_{i j}^{\mathrm{T}} \widehat{W}_{i j}\right) \leq-(1 / 2)\left\|\widetilde{W}_{i j}\right\|_{F}^{2}+(1 / 2)\left\|W_{i j}\right\|_{F}^{2} \\
z_{i j-1}^{\mathrm{T}} \tilde{\eta}_{i j} \leq(1 / 2)\left\|z_{i j-1}\right\|^{2}+(1 / 2)\left\|\tilde{\eta}_{i j}\right\|^{2} \\
\tilde{\eta}_{i j}^{\mathrm{T}} \pi_{i j} \leq\left(\pi_{i j}^{*} / 2 \Delta_{i j}\right)\left\|\tilde{\eta}_{i j}\right\|^{2}+\Delta_{i j} / 2
\end{array}\right.
$$

Make the following names.

$$
\left\{\begin{array}{l}
\epsilon_{i j}^{1}=\kappa_{i j}-\frac{1}{2}-\frac{\varepsilon_{i j}^{* 2}}{2 \varrho_{i j}}, \epsilon_{i q_{i}}^{1}=\kappa_{i q_{i}}-\frac{\varepsilon_{i q_{i}}^{*}}{2 \varrho_{i q_{i}}} \\
\epsilon_{i j}^{2}=\frac{\sigma_{i j}}{2}, \epsilon_{i j}^{3}=\frac{1}{\tau_{i j}}-\frac{1}{2}-\frac{\pi_{i j}^{* 2}}{2 \Delta_{i j}}
\end{array}\right.
$$

Then, $\dot{V}_{i}$ can be rewritten as

Where

$$
\dot{V}_{i} \leq-c_{i 0} V_{i}+c_{i 1}
$$

$$
\left\{\begin{array}{l}
c_{i 0}=\min \left\{2 \epsilon_{i j}^{1}, 2 \epsilon_{i j}^{2}, 2 \epsilon_{i j}^{3}\right\} \\
c_{i 1}=\sum_{j=1}^{q_{i}} \frac{\varrho_{i j}}{2}+\sum_{j=1}^{q_{i}} \frac{\sigma_{i j}\left\|w_{i j}\right\|_{F}^{2}}{2}+\sum_{j=2}^{q_{i}} \frac{\Delta_{i j}}{2}
\end{array}\right.
$$

Theorem 1. Consider the multi-agent system (1) in the strict-feedback form with stationary multi-targets. Suppose that Assumptions 1-3 hold. For any bounded initial condition $\mathrm{V}_{\mathrm{i}}(0) \leq \mu_{\mathrm{i}}$, if we choose design parameters satisfy $c_{i 0}>0$, then all agents will achieve the multi-target rotating encirclement formation with the proposed control scheme in Control Design.

Proof. By integrating both ends, it is obvious that the solution of (24) satisfies the following inequality.

$$
V_{i} \leq\left[V_{i}(0)-\frac{c_{i 1}}{c_{i 0}}\right] e^{-c_{i 0} t}+\frac{c_{i 1}}{c_{i 0}}
$$

Then, with Lemma 1 in Ref. 8, we know that the tracking error $z_{i 1}$ is bounded, and the upper bound is associated with $c_{i 1} / c_{i 0}$. By reasonably selecting design parameters, $z_{i 1}$ can be sufficiently reduced to 0 .

Combining with Lemma 1,2,3 and 4, we can easily conclude that our proposed control scheme will drive all agents achieve the multi-target rotating encirclement formation.

\section{Conclusion}

The collective multi-target rotating encirclement formation problem of strict-feedback multi-agent systems is investigated by dividing into three subproblems. Our proposed control scheme can solve this problem well.

\section{Acknowledgements}

This work was supported by the NSFC (61327807, 61521091, 61520106010, 61134005) and the National Basic Research Program of China (973 Program: 2012C B821200, 2012CB821201).

\section{References}

1. F. Chen, W. Ren and Y. Cao, Surrounding control in cooperative agent networks, Syst. Control Lett., 59(11), 2010, pp. 704-712.

2. P. Lin and Y. Jia, Distributed rotating formation control of multi-agent systems, Syst. Control Lett., 59(10), 2010, pp. 587-595.

3. M. Deghat, I. Shames, B.D.O. Anderson and C. Yu, Localization and circumnavigation of a slowly moving target using bearing measurements, IEEE T. Automat. Contr., 59(8), 2014, pp. 2182-2188.

4. L. Mo, X. Yuan and Q. Li, Finite-time rotating target-encirclement motion of multi-agent systems with a leader, Chinese J. Phys., 56(5), 2018, pp. 2265-2274.

5. T. Zhang, J. Ling and L. Mo, Distributed Finite-Time Rotating Encirclement Control of Multiagent Systems With Nonconvex Input Constraints, IEEE Access, 7, 2019, pp. $102477-102486$

6. R. Li, Y. Shi and Y. Song, Localization and circumnavigation of multiple agents along an unknown target based on bearing-only measurement: A three dimensional solution, Automatica, 94, 2018, pp. 18-25.

7. J. Shao and Y. Tian, Multi-target localisation and circumnavigation by a multi-agent system with bearing measurements in 2D space, Int. J. Syst. Sci., 49(1), 2018, pp. 15-26

8. T. Zhang and Y. Jia, Adaptive neural network control of uncertain strict-feedback systems with full-state constraints by integral-barrier Lyapunov functions, Proc. 37th Chin. Control Conf., Wuhan, China, 2018, pp. 846-851.

9. S.J. Yoo, Distributed consensus tracking of a class of asynchronously switched nonlinear multi-agent systems, Automatica, 87, 2018, pp. 421-427.

10. T. Zhang and Y. Jia, Adaptive neural network control of uncertain strict-feedback systems with full-state constrains by integral-barrier Lyapunov functions, Proc. 2018 Chin. Intel. Syst. Conf., Wenzhou, China, 2018, pp. 71-84.

11. W. He, S. Zhang and S.S. Ge, Adaptive control of a flexible crane system with the boundary output constraint, IEEE T. Ind. Electron., 61(8), 2014, pp. 4126-4133. 